\title{
Design of phase-only reconfigurable planar array antenna in selected phi cuts using various meta-heuristic optimization algorithms
}

\author{
D JAMUNAA $^{1}$, G K MAHANTI ${ }^{1, *}$ and FERAS NADHIM HASOON AL ATTAR ${ }^{2}$ \\ ${ }^{1}$ Department of Electronics and Communication Engineering, National Institute of Technology, \\ Durgapur 713209, India \\ ${ }^{2}$ Department of Electrical and Computer Engineering, College of Engineering, NUST, Muscat, Sultanate of \\ Oman \\ e-mail: gautammahanti@yahoo.com
}

MS received 5 July 2018; revised 11 December 2018; accepted 26 December 2018; published online 21 March 2019

\begin{abstract}
In this paper, a pattern synthesis based on a multiobjective optimization algorithm is proposed for the generation of a reconfigurable pencil/flat top dual-beam planar antenna array built using isotropic antenna elements in selected phi cuts. These beams claim the same amplitude excitations and differ from each other in phase excitations. Zero-phase excitations are used in pencil beam and these phases are updated with optimum phases for the flat top beam. All the excitations are obtained using Moth-flame optimization algorithm. With the support of the fitness functions, care is taken to control the expected values of the radiation pattern parameters to remain under certain fixed limit. In addition, synthesis is also done for the provision of a null in a particular direction for rejection of interference in the pencil beam in two different phi cuts. To suppress the mutual coupling effects, dynamic range ratio is kept under a threshold limit. Simulation results show the effectiveness of this proposed synthesis for phi cut planes. This algorithm is compared and proved to be better in many aspects over the standard meta-heuristic algorithms like Artificial Bee Colony and Imperialist Competitive algorithms in terms of performance parameters.
\end{abstract}

Keywords. Phase-only reconfigurable array antenna; Side Lobe Level; flat top beam; Moth-flame optimization algorithm; Artificial Bee Colony algorithm; Imperialist Competitive algorithm.

\section{Introduction}

Many of the telecommunication applications utilize reconfigurable antenna arrays in recent years because of their ability in generating different radiation patterns from a single antenna array. These array patterns are generated by switching between different phase excitations while sharing the same amplitude excitations. However, producing such kind of dual patterns involves quite a lot of challenges. Literature review reveals different approaches and methods that have been tested successfully in the past to different geometries of arrays. Out of available geometries of antenna arrays, planar arrays [1] have the advantages over individual antennas and other geometries in terms of providing high directional beams and symmetrical patterns with low Side Lobe Levels (SLL). They also have control in both the elevation and azimuthal planes. However, a closer look at the literature would reveal the concentration of generation of dual patterns mostly using linear arrays. To quote a few, Bucci et al [2] successfully synthesized various beam patterns using common amplitude and phase

*For correspondence shifters using the method of projection. Baskar et al [3] utilized a method based on improved multi-agent genetic algorithm and proved its effectiveness over other algorithms in terms of its solution quality and consistency. With the help of the control of non-uniform samples of the array factor, reconfigurable linear antenna array was generated successfully by Azevedo [4]. By controlling the phases, Buttazzoni and Vescovo [5] synthesized desired patterns using an iterative method and method of successive projections. Guo et al [6] presented two and three pattern beams by controlling the phases using the non-linear leastsquare method. Chatterjee et al [7] generated dual-beam pattern for a concentric ring array antenna in particular planes successfully using evolutionary algorithms. Many other methods are also reported in the literature [8, 9].

In addition to SLL in the patterns, there always exists a requirement of provision of null [10-15] to avoid interference or signals of nil interest in the patterns. Apart from the SLL and provision of null, necessary care is always taken to reduce the influence of mutual coupling, since mutual coupling plays a crucial role in the shape of the expected radiation patterns. This is done with the support of Dynamic Range Ratio (DRR). 
In this paper, two cases of a planar array with dual beams are dealt with. In case 1 , it is synthesized in various phi cut planes $\left(0^{\circ}, 60^{\circ}, 120^{\circ}, 180^{\circ}, 240^{\circ}\right.$ and $\left.300^{\circ}\right)$ simultaneously with restrictions on parameters, namely, SLL and ripple in flat top beam, and SLL in pencil beam. In case 2, the planar array is synthesized with a few more constraints on parameters that include SLL and a null position in the pencil beam, and SLL and ripple value in the flat top beam. This is done for two different values of azimuthal angles or phi cuts $\left(0^{\circ}\right.$ and $\left.10^{\circ}\right)$. Analysis is also done on the values of the beam parameters using random phi cuts between $0^{\circ}$ and $10^{\circ}$.

In both the syntheses, necessary care is taken to keep the amplitude as well as the phase excitations under certain limits. Both the beams share common amplitude excitations lying between 0 and 1 , whereas they differ in phase excitations lying between $-180^{\circ}$ and $180^{\circ}$. All the phase excitations are made equal to zero in the pencil beam and the evolutionary algorithms help in generating the phase excitations for the flat top beam in addition to the common amplitude excitations.

Moth-flame optimization (MFO) algorithm [16-18] is used in this synthesis process. It is duly compared to wellknown algorithms, namely Artificial Bee Colony algorithm (ABC) [19-21] and Imperialist Competitive algorithm (ICA) [22-24] for verifying its performance. The reason for using MFO algorithm is because of its success in solving benchmarked problems more comfortably over other algorithms. It is proved that it balances exploration and exploitation properly [16]. It also has the other following properties proved, namely fast convergence, high avoidance of local optima, secured best solutions and effectiveness in solving problems with unknown search spaces [16].

The novelty of this paper is that the radiation patterns are generated in various phi cut planes simultaneously with expected parameters using the same excitations in one of the synthesis. In the other synthesis, the values of the expected radiation parameters are made to fall under fixed desired limits for two phi cut planes with a provision for null. This also includes the values of the excitation amplitudes to satisfy the required DRR, which eliminates the impact of undesired mutual coupling. The reason for these syntheses is that this will suit applications like satellite and radar communications, where there is a requirement of dual beams, especially in a range of azimuthal planes.

\section{Problem formulation}

A planar array of isotropic elements is shown in figure 1. The far-field pattern $F F(\theta, \varphi)$ of this array consisting of $M$ elements in $x$-axis and $N$ elements in $y$-axis is given by Eq. (1):

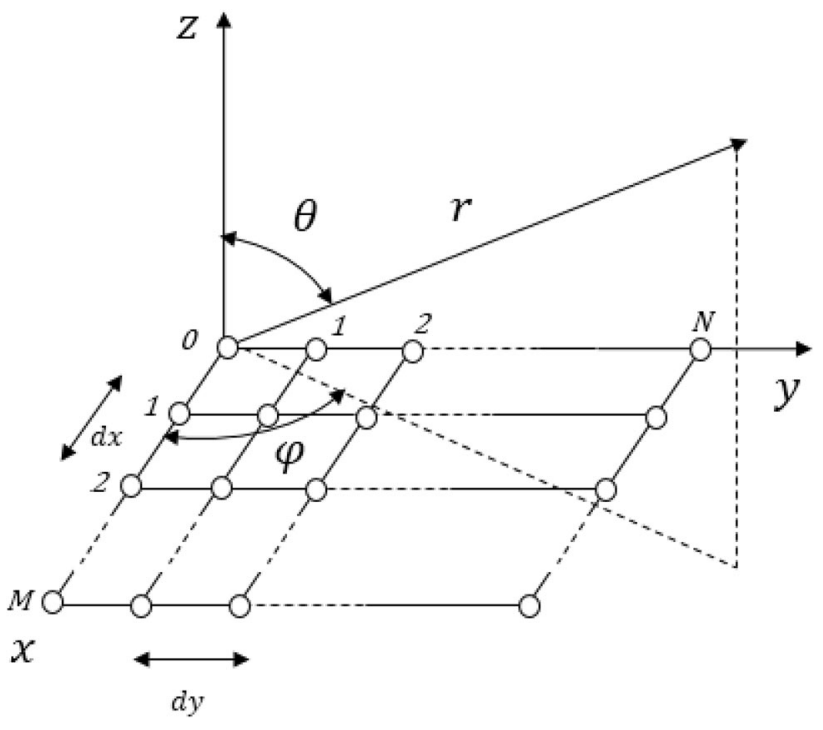

Figure 1. Geometry of a planar array.

$$
F F(\theta, \varphi)=\sum_{m=1}^{M} \sum_{n=1}^{N} C_{m n} e^{j\left(k m d_{x} \sin \theta \cos \varphi+k n d_{y} \sin \theta \sin \varphi+\rho_{m n}\right)}
$$

where $C_{m n}$ is the current amplitude excitation, $k$ is the wave number, $d_{x}$ and $d_{y}$ are the inter-element spacing $=0.5 \lambda$ and $\rho_{m n}$ is the phase excitation of the elements.

The algorithms are allowed to run based on the fitness functions that are necessary for generation of beams. The fitness or the cost function for this process is described as follows.

For case 1, the individual fitness function for the pencil beam pattern is given by

$$
\operatorname{Fitp}(\operatorname{exc})=w_{1}(S L L d p-S L L o p)^{2}
$$

where SLLdp and SLLop are, respectively, the desired and obtained values of SLL in $\mathrm{dB}$ and exc $=$ $\left\{C_{m n}, \rho_{m n}\right\}, 1 \leq m \leq M, 1 \leq n \leq N$ in the $M \times N$ array.

The individual fitness function for the flat top beam pattern is given by

$$
\operatorname{Fitf}(\operatorname{exc})=w_{2}(S L L d f-S L L o f)^{2}+w_{3}(R d f-R o f)^{2}
$$

where SLLdf and SLLof are, respectively, the desired and obtained values of SLL in $\mathrm{dB} ; R d f$ and $R o f$ are the desired and obtained values of ripple in $\mathrm{dB}$.

The total fitness for case 1 is given by

$$
F 1=F i t p(e x c)+F i t f(e x c) .
$$

For case 2, the fitness is given by

$$
F 2=F 1+w_{4}(\text { Nulldp }- \text { Nullop })^{2}+\left(D R R_{d}-D R R_{o}\right)^{2}
$$

where Nulldp and Nullop are, respectively, the desired and obtained values of null depth in $\mathrm{dB}$ for pencil beam; $D R R_{d}$ 
and $D R R_{o}$ are the desired and obtained values of $D R R$ referring to the ratio of the maximum to minimum value of amplitude excitations. If the obtained value is less than the desired value of any particular parameter, then the corresponding error will be zero. $F 1$ is written for various phi cut planes $\left(0^{\circ}, 60^{\circ}, 120^{\circ}, 180^{\circ}, 240^{\circ}\right.$ and $\left.300^{\circ}\right)$ and $F 2$ is written for two phi cut planes, namely at $\varphi=0^{\circ}$ and at $\varphi=10^{\circ}$ and optimization is carried out in one shot; $w_{1}-w_{4}$ are the weights associated with the parameters. The values of all the weights are kept to unity in both the cases except for $w_{3}=10$ in case 2 . All the desired parameter values are shown in tables 1 and 2 .

\section{MFO algorithm}

The methods of obtaining the solutions to very complex optimization problems relied much on the evolutionary algorithms in the past few decades. This paper utilizes the MFO algorithm, a recent meta-heuristic one developed by Mirjalili [16]. This algorithm takes into consideration the navigation principles of moths with reference to transverse orientation. These moths fly in night while maintaining a certain angle with respect to moon. This is an effective mechanism to travel long distance in a straight path. However, the same is not possible if the distance is very

Table 1. Desired and obtained values for pencil and flat top beam patterns for case 1 .

\begin{tabular}{|c|c|c|c|c|}
\hline \multirow[b]{2}{*}{$\varphi(\operatorname{deg})$} & \multirow{2}{*}{$\begin{array}{c}\text { Beams } \\
\text { parameters } \\
\text { desired value }\end{array}$} & \multirow{2}{*}{$\begin{array}{c}\text { Pencil beam } \\
\text { SLL }(\mathrm{dB}) \\
-17\end{array}$} & \multicolumn{2}{|c|}{ Flat top beam } \\
\hline & & & $\begin{array}{l}\text { SLL }(d B) \\
-17\end{array}$ & $\begin{array}{c}\text { Ripple value }(\mathrm{dB}) \\
1\end{array}$ \\
\hline \multirow[t]{3}{*}{$\overline{0}$} & ICA & -17.0142 & -16.9997 & 1.1713 \\
\hline & $\mathrm{ABC}$ & -17.3970 & -15.7389 & 2.7539 \\
\hline & MFO & -16.9927 & -16.9890 & 1.0426 \\
\hline \multirow[t]{3}{*}{60} & ICA & -17.0112 & -16.9964 & 1.1713 \\
\hline & $\mathrm{ABC}$ & -18.2111 & -15.5903 & 2.7296 \\
\hline & MFO & -17.9416 & -17.0186 & 1.0430 \\
\hline \multirow[t]{3}{*}{120} & ICA & -20.8611 & -16.9982 & 1.1693 \\
\hline & $\mathrm{ABC}$ & -21.0049 & -15.7655 & 2.5790 \\
\hline & MFO & -16.9941 & -16.9971 & 1.0312 \\
\hline \multirow[t]{3}{*}{180} & ICA & -17.0142 & -17.0033 & 1.1710 \\
\hline & $\mathrm{ABC}$ & -17.3970 & -15.7389 & 2.1023 \\
\hline & MFO & -16.9927 & -16.9984 & 0.7628 \\
\hline \multirow[t]{3}{*}{240} & ICA & -17.0112 & -16.9964 & 1.1714 \\
\hline & $\mathrm{ABC}$ & -18.2111 & -15.5842 & 2.0712 \\
\hline & MFO & -17.9416 & -17.0186 & 1.0430 \\
\hline \multirow[t]{3}{*}{300} & ICA & -20.8611 & -16.9979 & 1.0211 \\
\hline & $\mathrm{ABC}$ & -21.0049 & -15.7655 & 2.2386 \\
\hline & MFO & -16.9941 & -16.9971 & 1.0429 \\
\hline
\end{tabular}

Table 2. Desired and obtained values for pencil and flat top beam patterns for case 2 .

\begin{tabular}{|c|c|c|c|c|c|c|c|c|}
\hline \multirow{3}{*}{$\begin{array}{l}\varphi \\
(\operatorname{deg})\end{array}$} & \multirow{3}{*}{$\begin{array}{c}\text { Parameters }(\mathrm{dB}) \text { (desired } \\
\text { value) }\end{array}$} & \multicolumn{3}{|c|}{ Pencil beam pattern values $(\mathrm{dB})$} & \multirow{3}{*}{$\begin{array}{l}\text { Parameters }(\mathrm{dB}) \\
\text { (desired value) }\end{array}$} & \multicolumn{3}{|c|}{ Flat top beam pattern values $(\mathrm{dB})$} \\
\hline & & \multicolumn{3}{|c|}{ Obtained } & & \multicolumn{3}{|c|}{ Obtained } \\
\hline & & MFO & $\mathrm{ABC}$ & ICA & & MFO & $\mathrm{ABC}$ & ICA \\
\hline \multirow[t]{2}{*}{0} & SLL $(-20 \mathrm{~dB})$ & -20.0938 & -20.0794 & -19.5799 & SLL $(-20 \mathrm{~dB})$ & -21.3847 & -20.2136 & -20.0134 \\
\hline & $\begin{array}{l}\text { Null depth at } \theta=-60^{\circ}(- \\
45 \mathrm{~dB})\end{array}$ & -45.8309 & -51.6725 & -45.7994 & Ripple (1 dB) & 0.7732 & 0.9793 & 1.0719 \\
\hline \multirow[t]{2}{*}{10} & SLL $(-20 \mathrm{~dB})$ & -21.2069 & -20.0930 & -20.4630 & SLL $(-20 \mathrm{~dB})$ & -22.2277 & -20.0266 & -19.898 \\
\hline & $\begin{array}{l}\text { Null depth at } \theta=-60^{\circ}(- \\
45 \mathrm{~dB})\end{array}$ & -48.0432 & -49.8743 & -54.6615 & Ripple (1 dB) & 0.9117 & 0.9762 & 1.0718 \\
\hline DRR & 6 & 5.7013 & 6.0984 & 6.4109 & 6 & 5.7013 & 6.0984 & 6.4109 \\
\hline \multicolumn{2}{|c|}{ Fitness value } & 0 & 0.009532 & 0.409054 & 0 & 0 & 0.009532 & 0.409054 \\
\hline \multicolumn{2}{|c|}{$\begin{array}{l}\text { Iterations for fitness values to } \\
\text { reach } 0\end{array}$} & 448 & - & - & - & 448 & - & - \\
\hline
\end{tabular}


less and moreover, these flies get trapped in an unwanted spiral path when they get attracted towards artificial lights.

In this algorithm [16-18], the moths are considered as the solutions (amplitudes and phases) and the positions of the moths as variables used in the problem. Moreover, both the moths and the flames are treated as solutions. The difference between the moths and the flames is how they are treated and updated after every iteration. The moths are the search agents that travel around the search space and flames are the best positions of moths that are obtained till that time. Hence, each moth searches around a flame and updates it when a better solution is found. Hence, in any case, a moth will never lose its best solution found. The update mechanism of moths used is that of a logarithmic spiral. The following points should be considered when any other type of spiral is used. The initial point of the spiral should be the moth itself and the final point should be the flame. Any fluctuation of the spiral range should be within the search space considered.

The logarithmic spiral for the MFO algorithm is as follows:

$$
S\left(M_{x}, F_{x}\right)=D i s_{x} e^{c t} \cos (2 \pi t)+F_{y}
$$

where $D i s_{x}=\left|F_{y}-M_{x}\right|$ refers to the distance of the $x^{\text {th }}$ moth for the $y^{\text {th }}$ flame, $F_{y}$ is the $y^{\text {th }}$ flame, $M_{x}$ is the $x^{\text {th }}$ moth, $c$ is a constant defining the shape of the spiral and $t$ is a random number between -1 and 1 .

From Eq. (6), it is seen that the next position is defined with respect to a flame. The random number $t$ in $[r, 1]$ defines the closeness of the next position of the moth to the flame. The term $r$ is the adaptive convergence constant. This $r$ is linearly decreased from -1 to -2 over the course of iteration and helps in increasing the speed of convergence. Moreover, this equation also allows the moth to fly around flames and it need not travel necessarily in the space between them. This increases the exploration and exploitation capability of the search space. The matrix $F$ also includes the recent best solutions obtained till any iteration. The moths update their positions with respect to this matrix during the optimization process.

In order to prevent any fall in local optima, each moth is made to update its position using only one of the flames. After each and every iteration, once the list of flames is updated, the flames are ranked based on the fitness values. The moths then update the positions with respect to the flames. The first moth updates its position with respect to the best flame and the last moth with respect to the worst flame.

Another concern is that the position updating of moths with respect to different locations in the search space may end up in degradation of the best promising solutions. To avoid this, the following formula is used:

$$
\text { flame number }=\text { round }\left(N-l \times \frac{N-1}{T}\right)
$$

where $l$ is the current number of iteration, $N$ is the maximum number of flames and $T$ is the maximum number of iterations. The moths update their positions only with respect to the best flame in the final steps of given iterations. The gradual decrease in number of flames balances exploration and exploitation of the search space [16].

The exploration capability is guaranteed because of the following reasons.

Moths update their positions in hyper-spheres around the best solutions obtained so far.

The flame sequences are modified based on the best obtained solutions in each iteration. Therefore, the updating of the positions of the moths occurs around different flames, which can cause sudden movement of moths in search space.

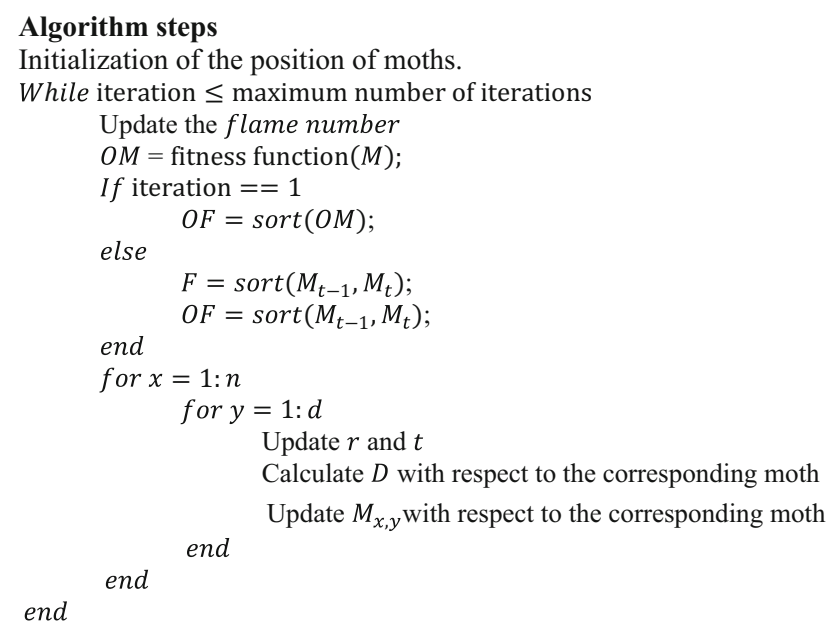

\section{Simulated results}

\subsection{Parameter settings}

A $7 \times 7$ planar array consisting of 49 elements is chosen for simulation purposes. The spacing between the elements, namely $d_{x}$ and $d_{y}$, is chosen to be $0.5 \lambda$. In case 1 , synthesis is done for various phi cut planes $\left(0^{\circ}, 60^{\circ}, 120^{\circ}\right.$, $180^{\circ}, 240^{\circ}$ and $300^{\circ}$ ) at a time. All the algorithms are run for a maximum of 2000 iterations and the results are presented from the best of 10 runs for all the MFO, ABC and ICA algorithms. All the weights associated with the parameters are kept equal to unity. For ABC algorithm, the food number is kept at $0.5 \times$ population size and the limit is kept at 30 for a food source that could not be improved through "limit" trials. For MFO algorithm, $N=$ population size in Eq. (7). For ICA algorithm, number of empires $=98$, selection pressure $=1$, assimilation coefficient $=1$, revolution probability $=0.05$ and revolution rate $=0.1$ are used. MATLAB software is used for the simulation purposes. 
Table 1 shows the necessary specifications for both the pencil and flat top beam patterns along with the obtained values using both for case 1 . Figure $2 \mathrm{a}$ and $\mathrm{b}$ shows the amplitude and phase distributions generated by MFO algorithm. Two sample figures out of 6 various phi cut planes are shown in this paper. Figures 3 and 4 show normalized power in $\mathrm{dB}$ vs. $\theta$ in degrees with $\varphi=0^{\circ}$ and

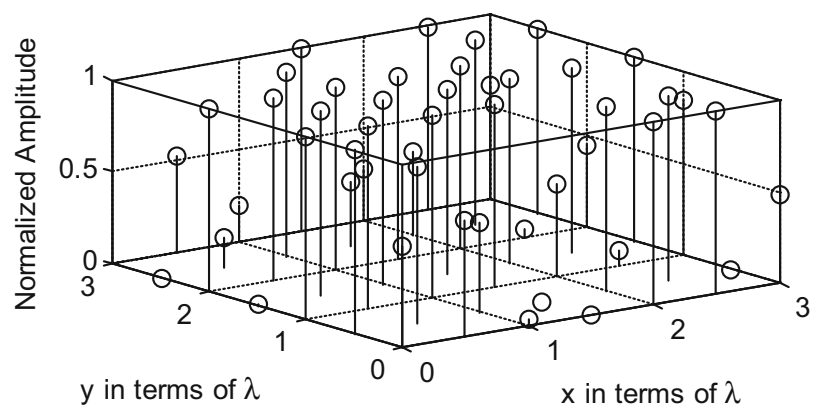

(a) Normalized amplitude excitations obtained using MFO algorithmfor case 1.

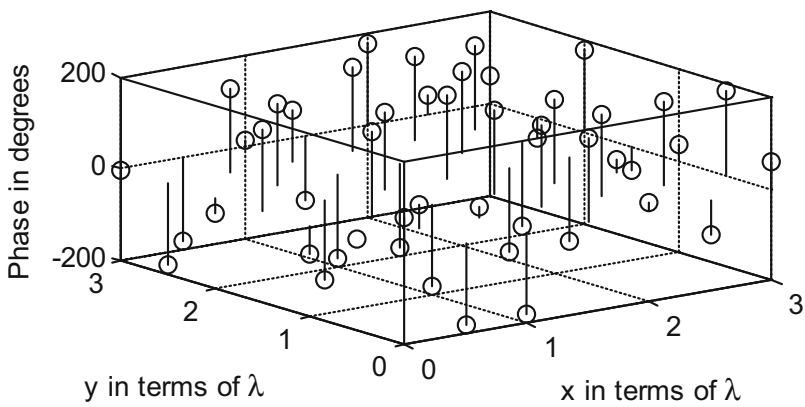

(b) Phase excitations obtained using MFO algorithm for case 1 .

Figure 2. (a) Normalized amplitude excitations obtained using MFO algorithm for case 1. (b) Phase excitations obtained using MFO algorithm for case 1 .

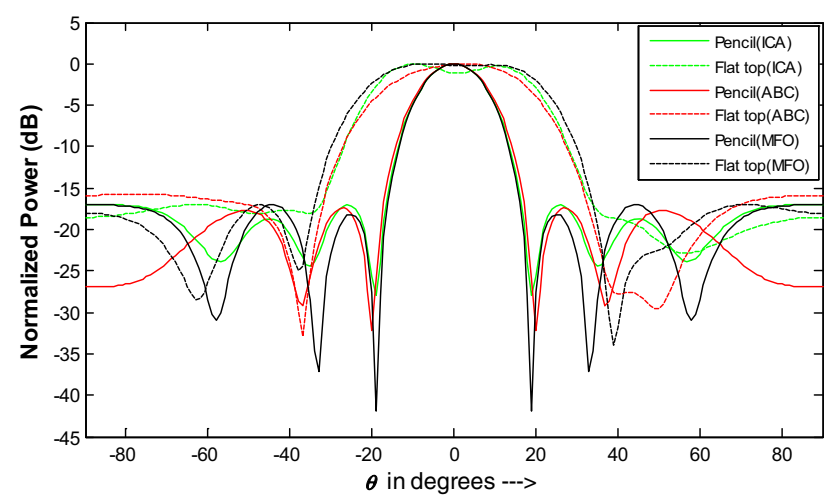

Figure 3. Normalized power pattern in $\mathrm{dB}$ vs. $\theta$ in degrees at $\varphi=0^{\circ}$ using ICA, ABC and MFO algorithms for case 1 .

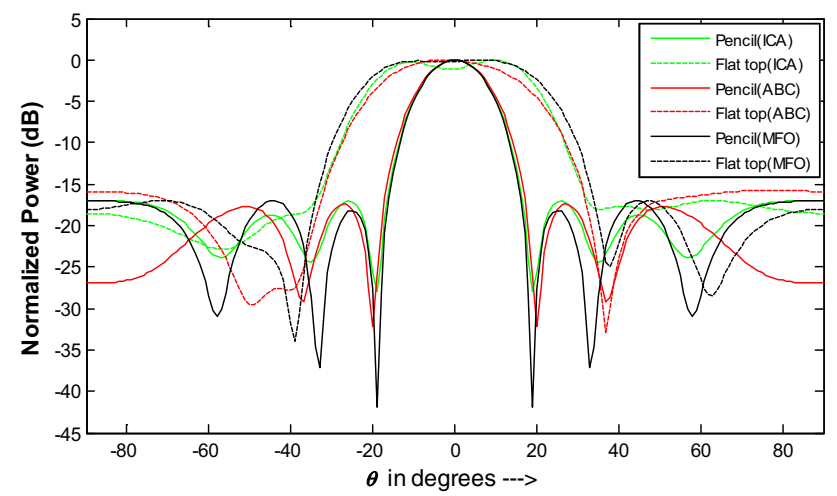

Figure 4. Normalized power pattern in $\mathrm{dB}$ vs. $\theta$ in degrees at $\varphi=180^{\circ}$ using ICA, ABC and MFO algorithms for case 1 .

$\varphi=180^{\circ}$, respectively, using ICA, ABC and MFO algorithms for case 1 .

\subsection{Analysis for case 1}

Table 1 shows that even though MFO and ICA algorithms have produced values near the expected SLL values in both beams, MFO has produced better results at most of the

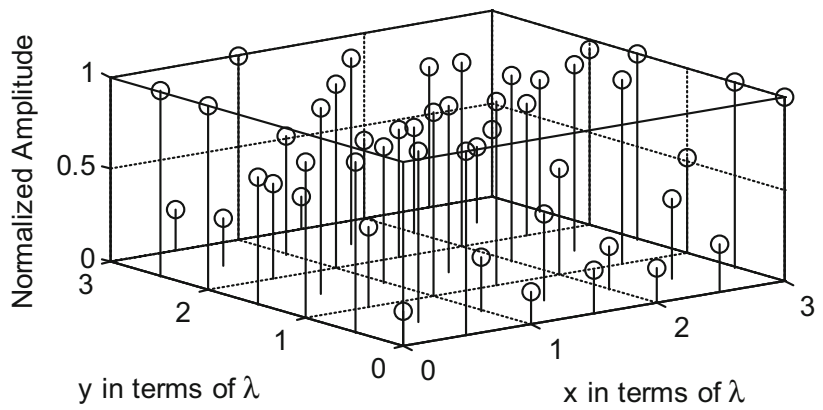

(a) Normalized amplitude excitations obtained using MFO algorithm for case 2.

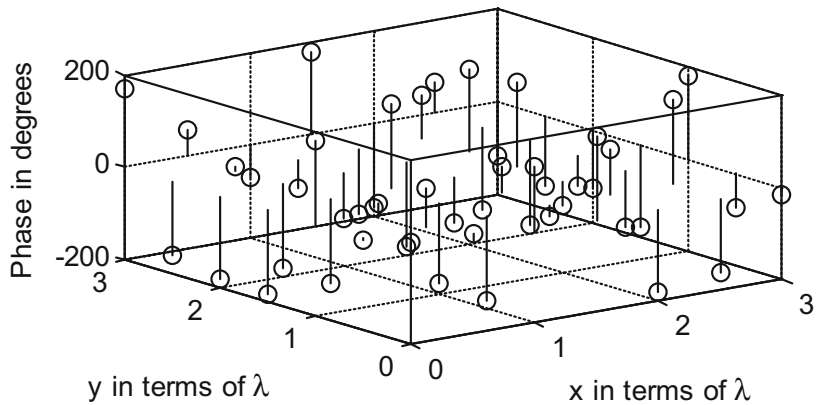

(b) Phase excitations obtained using MFO algorithm for case 2 .

Figure 5. (a) Normalized amplitude excitations obtained using MFO algorithm for case 2. (b) Phase excitations obtained using MFO algorithm for case 2 . 
angles. It also shows that MFO has clearly produced the lowest ripple value in $\mathrm{dB}$ for all the various phi cut planes over the other two algorithms. It even produced a ripple value of $0.7628 \mathrm{~dB}$, but all the other values are more than $1 \mathrm{~dB}$.

For case 2, syntheses of the expected beams are done and the radiation pattern is evaluated at only two cut planes, namely at $\varphi=0^{\circ}$ and at $\varphi=10^{\circ}$, with restrictions on DRR of 6 . All the weights associated with the parameters are kept equal to unity except $w_{3}$, which is kept at 10 . The primary reason for this is to keep the ripple value less than the required one in order to maintain the shape of the flat

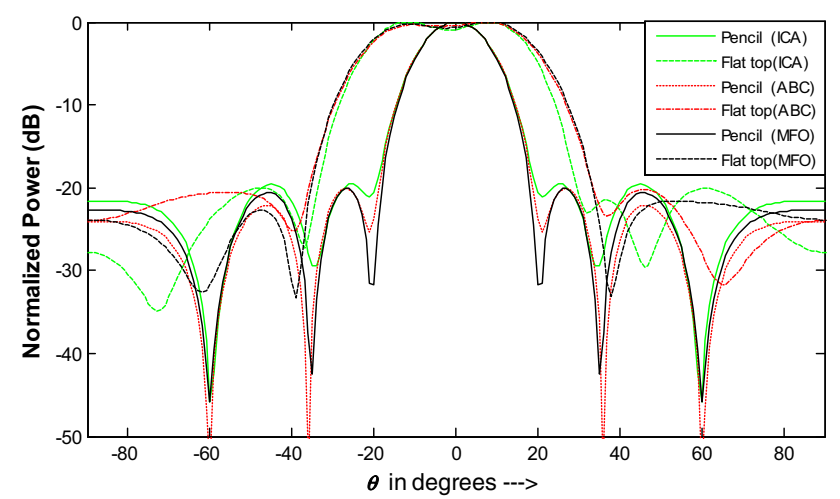

Figure 6. Normalized power pattern in $\mathrm{dB}$ vs. $\theta$ in degrees at $\varphi=0^{\circ}$ using ICA, ABC and MFO algorithms for case 2.

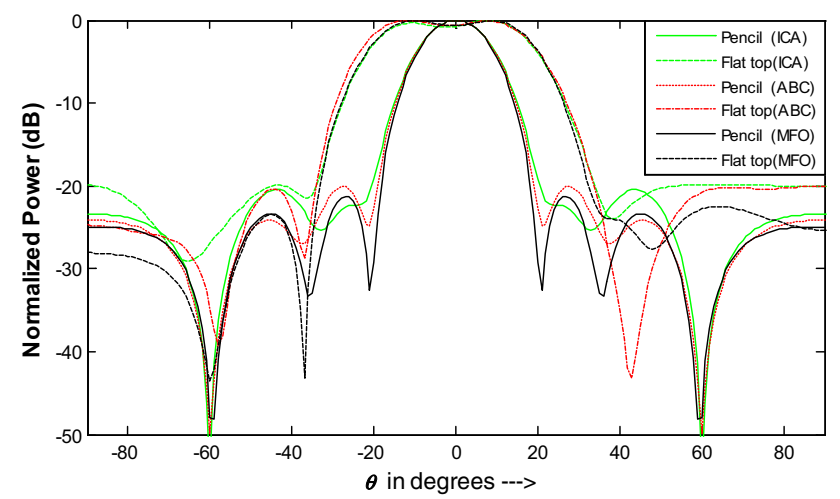

Figure 7. Normalized power pattern in $\mathrm{dB}$ vs. $\theta$ in degrees at $\varphi=10^{\circ}$ using ICA, ABC and MFO algorithms for case 2. top beam. This is done because it is found to be difficult to maintain this value in case 2 with unity weight.

Table 2 gives the results of both the desired and obtained values using all the algorithms for the pencil as well as the flat top beam patterns. Figure $5 \mathrm{a}$ and $\mathrm{b}$ shows the amplitude and phase excitations obtained using MFO algorithm and figures 6 and 7 show the radiation patterns using all the algorithms.

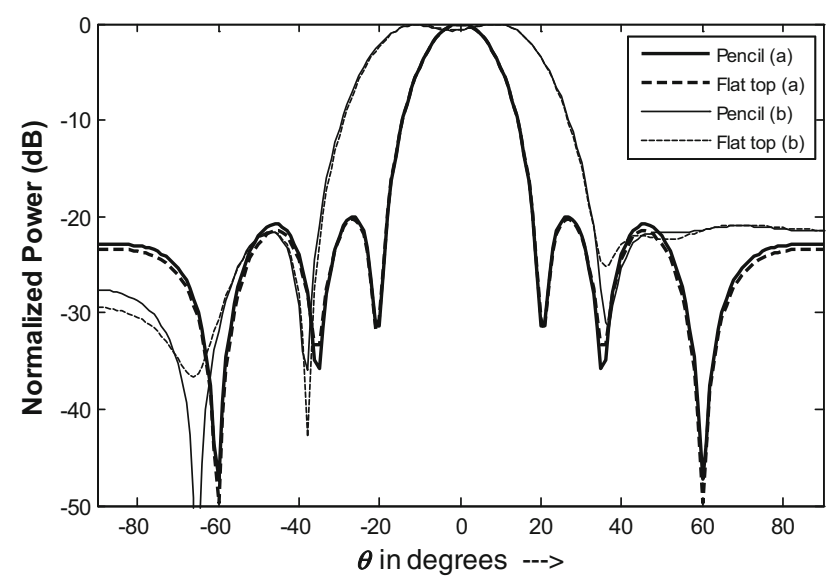

Figure 8. Normalized power pattern in $\mathrm{dB}$ vs. $\theta$ in degrees at random cut planes using MFO for case 2 ; (a) refers to $\varphi=3^{\circ}$ and (b) refers to $\varphi=6^{\circ}$.

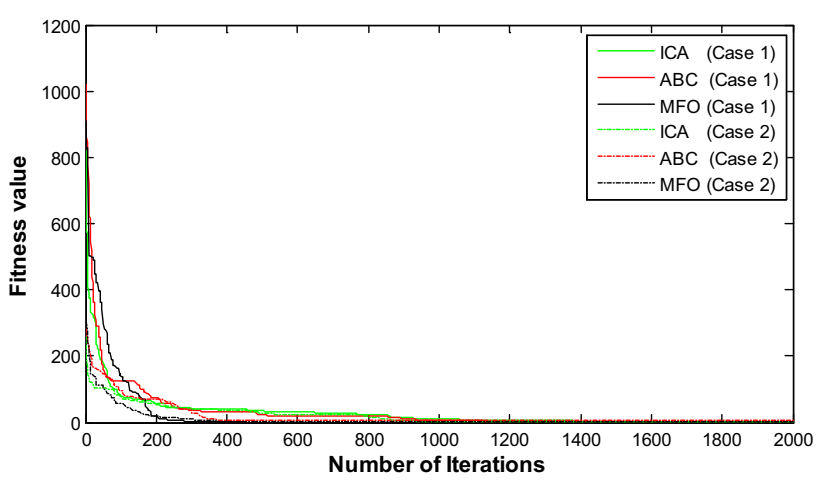

Figure 9. Fitness values vs. number of iterations.

Table 3. Desired and obtained values at random cut planes using MFO for case 2 .

\begin{tabular}{|c|c|c|c|c|c|c|}
\hline \multirow[b]{2}{*}{$\varphi(\operatorname{deg})$} & \multirow[b]{2}{*}{ Parameters (dB) } & \multicolumn{2}{|c|}{ Pencil beam pattern values $(\mathrm{dB})$} & \multirow[b]{2}{*}{ Parameters $(\mathrm{dB})$} & \multicolumn{2}{|c|}{ Flat top beam pattern values $(\mathrm{dB})$} \\
\hline & & Desired & Obtained & & Desired & Obtained \\
\hline \multirow[t]{2}{*}{3} & Side lobe level & -20 & -20.0315 & Side lobe level & -20 & -20.9883 \\
\hline & Null depth at $\theta=-60^{\circ}$ & -45 & -47.2098 & Ripple & 1 & 0.7200 \\
\hline \multirow[t]{2}{*}{6} & Side lobe level & -20 & -20.2921 & Side lobe level & -20 & -20.8619 \\
\hline & Null depth at $\theta=-60^{\circ}$ & -45 & -49.6935 & Ripple & 1 & 0.7604 \\
\hline
\end{tabular}


Table 4. Statistical parameters.

\begin{tabular}{|c|c|c|c|c|c|c|}
\hline \multirow[b]{2}{*}{ Statistical parameters out of 10 runs } & \multicolumn{3}{|c|}{ Case 1} & \multicolumn{3}{|c|}{ Case 2} \\
\hline & ICA & $\mathrm{ABC}$ & MFO & ICA & $\mathrm{ABC}$ & MFO \\
\hline Best fitness value & 0.0294 & 5.0805 & 0.0020 & 0.409054 & 0.009532 & 0 \\
\hline Mean & 4.8034 & 21.3485 & 5.6556 & 8.2738 & 3.4532 & 3.1699 \\
\hline Standard deviation & 2.8685 & 8.6496 & 5.2380 & 5.9188 & 3.5735 & 4.5633 \\
\hline Highest fitness value & 9.3469 & 37.0220 & 13.9765 & 18.0118 & 12.7003 & 12.3986 \\
\hline
\end{tabular}

\subsection{Analysis for case 2}

A look at figures 6 and 7 and the corresponding table 2 shows that MFO has outperformed ABC and ICA algorithms. At both $\varphi=0^{\circ}$ and $\varphi=10^{\circ}$ in the pencil beam pattern, the values of all the parameters obtained using MFO and ABC are well within the expected/desired values. However, the SLL in pencil beam $\left(\varphi=0^{\circ}\right)$ and flat top beam $\left(\varphi=10^{\circ}\right)$, ripple values in both $\varphi=0^{\circ}$ and $\varphi=10^{\circ}$ produced by ICA algorithm are not in compliance with the desired limit. Moreover, the values that are obtained for MFO algorithm are under the strict restriction of DRR being less than 6 , whereas its value is more than 6 using $\mathrm{ABC}$ and ICA algorithms.

MFO has also excelled over both of the other algorithms in terms of convergence speed. It took only 448 iterations to reach zero fitness value for MFO, whereas it never reached zero for the other algorithms.

To further analyse the performance of MFO algorithm, random cut planes are chosen, in this case at $\varphi=3^{\circ}$ and at $\varphi=6^{\circ}$, and the values of the obtained parameters are analysed. They are shown in table 3 . Figure 8 shows the radiation patterns obtained at different cut planes.

From table 3, it can be seen that there is no deviation between the desired and obtained values at random cut planes lying between $\varphi=0^{\circ}$ and $\varphi=10^{\circ}$, namely $\varphi=3^{\circ}$ and $\varphi=6^{\circ}$. In fact, the desired values completely match the flat top beam as well as the pencil beam pattern values. This further shows that the beam pattern evaluated at any angle between $0^{\circ}$ and $10^{\circ}$ does not deviate from the expected ones. Both of the power patterns obtained at random cut planes are shown in figure 8 and hence this analysis also confirms that the resultant pencil/flat top beam pair is not restricted to any single $\varphi$-cut of the individual beam patterns. Figure 9 shows a plot between fitness values and number of iterations and table 4 shows the statistical parameters.

Figure 9 clearly shows that the convergence of MFO algorithm is very fast; it reached 0 within very less number of iterations when compared with the remaining algorithms. From table 4, it is clearly proven that MFO has succeeded in the comparison dealing with convergence and best fitness value in both the cases. Mean and standard deviation values are found to be good in case 2. In case 1, ICA has produced best values in all the values except the most required best fitness value. Thus, it is clearly demonstrated that MFO has performed better than the other algorithms in terms of satisfying the constraint of DRR, radiation parameter values and best fitness values.

\section{Conclusion}

This paper presented a study on the design of phase-only reconfigurable antennas using evolutionary algorithms and it concentrated on the generation of expected radiation pattern parameters in selected phi-cuts. It also demonstrated the provision of a null in the pencil beam as well as ripple in the flat top beam along with SLL. The DRR was consideredin both the beams. A concerted effort was taken to converge the parameter values to the expected values for various cut planes within a limited range of azimuthal angles. MFO algorithm succeeded in providing the solutions successfully for the designed fitness functions and this procedure can be extended to other geometries of antenna arrays.

\section{References}

[1] Elliott R S 2003 Antenna theory and design. New York: Wiley Interscience

[2] Bucci O M, Mazzarella G and Panariello G 1991 Reconfigurable arrays by phase-only control. IEEE Transactions on Antennas and Propagation 39(7): 919-925

[3] Baskar S, Alphones A and Suganthan P N 2005 Genetic algorithm based design of a reconfigurable antenna array with discrete phase shifter. Microwave and Optical Technology Letters 45(6): 461-465

[4] Azevedo J A R 2008 Shaped beam pattern synthesis with non-uniform sample phases. Progress in Electromagnetics Research B 5: 77-90

[5] Buttazzoni G and Vescovo R 2012 Power synthesis for reconfigurable arrays by phase-only control with simultaneous dynamic range ratio and nearfield reduction. IEEE Transactions on Antennas and Propagation AP-60(2): 1161-1165

[6] Guo H, Guo C J, Qu Y and Ding J 2013 Pattern synthesis of phase-only reconfigurable linear arrays by nonlinear leastsquare method. In: Proceedings of the IEEE International Conference of IEEE Region 10

[7] Chatterjee A, Mahanti G K and Mahapatra P R S 2011 Design of fully digital controlled reconfigurable dual-beam 
concentric ring array antenna using gravitational search algorithm. Progress in Electromagnetics Research $C$ 18: $59-72$

[8] Mahanti G K, Chakraborty A and Das S 2007 Design of fully digital controlled reconfigurable array antennas with fixed dynamic range ratio. Journal of Electromagnetic Waves and Applications 21(1): 97-106

[9] Morabito A F, Massa A, Rocca P and Isernia T 2012 An effective approach to the synthesis of phase-only reconfigurable linear arrays. IEEE Transactions on Antennas and Propagation 60(8): 3622-3631

[10] Aksoy E and Afacan E 2009 Planar antenna pattern nulling using differential evolution algorithm. International Journal of Electronics and Commun (AEÜ) 6: 116-122

[11] Steyskal H 1982 Synthesis of antenna pattern with prescribed nulls. IEEE Transactions on Antennas and Propagation 30: 273-279

[12] Haupt R L 1997 Phase-only adaptive nulling with a genetic algorithm. IEEE Transactions on Antennas and Propagation 45: 1009-1015

[13] Liao W P and Chu F L 1999 Array pattern synthesis with null steering using genetic algorithms by controlling only the current amplitudes. International Journal of Electronics 86: $445-457$

[14] Chung Y C and Haupt R L 2000 Amplitude and phase adaptive nulling with a genetic algorithm. Journal of Electromagnetic Waves and Applications 14: 631-649

[15] Lozano M V, Rodriguez J A and Ares F 1999 Recalculating linear array antennas to compensate for failed elements while maintaining fixed nulls. Journal of Electromagnetic Waves and Applications 13: 397-412

[16] Mirjalili S 2015 Moth-flame optimization algorithm: a novel nature-inspired heuristic paradigm. Knowledge-Based Systems 89: 228-249
[17] Bahrami M, Bozorg-Haddad O and Chu X 2018 MothFlame Optimization (MFO) algorithm. In: Bozorg-Haddad $\mathrm{O}$ (Eds) Advanced Optimization by Nature-Inspired Algorithms. Studies in Computational Intelligence, vol. 720

[18] Buch H, Trivedi I N, Jangir P and Zheng P 2017 Moth flame optimization to solve optimal power flow with non-parametric statistical evaluation validation. Cogent Engineering 4: 1

[19] Karaboga D and Basturk B 2007 Artificial Bee Colony (ABC) optimization algorithm for solving constrained optimization problems. LNCS: Advances in Soft Computing: Foundations of Fuzzy Logic and Soft Computing, vol. 4529, pp. 789-798. Springer-Verlag, Berlin, Heidelberg

[20] Karaboga D and Basturk B 2007 A powerful and efficient algorithm for numerical function optimization: Artificial Bee Colony (ABC) algorithm. Journal of Global Optimization 39(3): 459-471

[21] Kumar A, Kumar D and Jarial S K 2017 A review on artificial bee colony algorithms and their applications to data clustering. Cybernetics and Information Technologies 17(3): $3-28$

[22] Abdollahi M, Isazadeh A and Abdollahi D 2013 Imperialist competitive algorithm for solving systems of nonlinear equations. Computers \& Mathematics with Applications 65(12): 1894-1908

[23] Bernal E, Castillo O, Soria J and Valdez F 2017 Imperialist competitive algorithm with dynamic parameter adaptation using fuzzy logic applied to the optimization of mathematical functions. Algorithms 10(1): 18

[24] Ji X, Gao Q, Yin F and Guo H 2016 An efficient imperialist competitive algorithm for solving the QFD decision problem. Mathematical Problems in Engineering 2016, Article ID 2601561, 13 pages 